\title{
Context effects in haptic perception of roughness
}

\author{
Mirela Kahrimanovic - Wouter M. Bergmann Tiest · \\ Astrid M. L. Kappers
}

Received: 2 October 2008/ Accepted: 19 December 2008/Published online: 21 January 2009

(C) The Author(s) 2009. This article is published with open access at Springerlink.com

\begin{abstract}
The influence of temporal and spatial context during haptic roughness perception was investigated in two experiments. Subjects examined embossed dot patterns of varying average dot distance. A two-alternative forcedchoice procedure was used to measure discrimination thresholds and biases. In Experiment 1, subjects had to discriminate between two stimuli that were presented simultaneously to adjacent fingers, after adaptation of one of these fingers. The results showed that adaptation to a rough surface decreased the perceived roughness of a surface subsequently scanned with the adapted finger, whereas adaptation to a smooth surface increased the perceived roughness (i.e. contrast after effect). In Experiment 2, subjects discriminated between subsequent test stimuli, while the adjacent finger was stimulated simultaneously. The results showed that perceived roughness of the test stimulus shifted towards the roughness of the adjacent stimulus (i.e. assimilation effect). These contextual effects are explained by structures of cortical receptive fields. Analogies with comparable effects in the visual system are discussed.
\end{abstract}

Keywords Tactile - Temporal adaptation . Spatial induction $\cdot$ After effect $\cdot$ Assimilation

M. Kahrimanovic $(\square) \cdot$ W. M. Bergmann Tiest .

A. M. L. Kappers

Physics of Man, Universiteit Utrecht,

Padualaan 8, $3584 \mathrm{CH}$ Utrecht, The Netherlands

e-mail: m.kahrimanovic@uu.nl

\section{Introduction}

Relevant information that we receive from our environment must be processed while a large amount of irrelevant information stimulates our senses. The concept of how contextual information influences perception is important for the study of perception and cognition. The large number of studies concerning contextual influences on perception in, for example, the visual domain emphasizes the importance of this concept (e.g. Adelson 1993; Cao and Shevell 2005; Ware and Cowan 1982; Webster et al. 2002). In the haptic modality, this concept has received less attention. However, during daily exploration by touch, we often perceive a particular object after having been in contact with some other object(s), or we explore different materials with different parts of the hand at the same time. Hence, the context in which haptic perception takes place may influence the perceptual experience. The current study was designed to investigate these contextual influences in the haptic perception of textured surfaces. These influences can roughly be subdivided into temporal and spatial influences of the context.

Temporal context

Roughness is one texture property that has been studied in some detail. Tactile roughness perception has been related to physical characteristics of the surface, like the spacing between and the height of surface elements (e.g. Connor et al. 1990; Connor and Johnson 1992; Lederman 1981; Lederman 1983; Lederman and Taylor 1972). Furthermore, studies addressing the neural codes underlying the sensation of tactile roughness showed that subjective roughness is related to spatial variations in the firing rate of slowly adapting type I (SAI) mechanoreceptive 
neurons (Blake et al. 1997; Connor et al. 1990; Connor and Johnson 1992).

Some ideas about the influence of temporal context in the perception of textured surfaces can be deduced from studies investigating the contribution of vibratory adaptation to roughness perception. Lederman et al. (1982) showed that the perceived magnitude of supraliminal vibrotactile signals decreased after adaptation to vibrations. More recently, Hollins et al. (2001) found that adaptation to vibrotactile signals disrupted the discrimination of very fine textured surfaces (spatial period $<200 \mu \mathrm{m}$ ). Furthermore, it has been shown that this type of adaptation had no effect on roughness perception of coarse surfaces, like metal gratings (Lederman et al. 1982) and dotted patterns with spatial periods above $200 \mu \mathrm{m}$ (Hollins et al. 2001). Also, when adapting to a spatially textured surface instead of vibrotactile stimuli, no adaptation effects with coarse surfaces were found (Hollins et al. 2006).

However, DiCarlo et al. (1998) suggested that texture adaptation effects should be present in the case of coarse surfaces. They used random dot stimuli to study the structure of receptive fields in area $3 \mathrm{~b}$ of the somatosensory cortex. The results revealed that most of these receptive fields have one or two inhibitory regions flanking a region of excitation. This resembles structures in the primary visual cortex, where many simple cells also have receptive fields with an excitatory region surrounded by flanking inhibitory areas (Hubel and Wiesel 1962). If the structures are highly similar, then this could indicate that cells from different brain areas represent information in analogous ways. Visual cortex cells are highly susceptible to adaptation (Blakemore et al. 1973; Jones and Palmer 1987). Adaptation causes a shift in the neuronal tuning of these visual neurons away from the level of the adapted value. Examples of such shifts have been found for dimensions like contrast (Carandini et al. 1997) and orientation (Dragoi et al. 2000).

Hence, if analogous processing of information occurs within different modalities, it should be expected that somatosensory and visual neurons should also show comparable adaptation effects. Consequently, texture adaptation should influence the perceived roughness of coarse surfaces, in apparent contrast to what Hollins et al. (2006) found. Their stimuli consisted of regular dot patterns with relatively small distances between dots, whereas DiCarlo et al. (1998) used random dot patterns with much larger average distances between dots. It could be that the regular patterns with smaller dot distances are not appropriate for activating the neuron types described by Dicarlo et al. (1998); therefore, no adaptation effects were found in the Hollins et al. (2001)'s study. Another possibility is that the adaptation pattern used by Hollins was too weak to cause significant adaptation effects.
In the first experiment of the current work, we used random dot patterns with relatively large average distances between dots and assumed that they will appropriately activate the neurons that are susceptible to adaptation. Subjects were asked to discriminate between the roughness of two surfaces presented simultaneously to two adjacent fingers, after adapting one of these fingers to a textured surface. It is hypothesized that texture adaptation will change the response patterns of neurons, resulting in changed perceived roughness of a subsequently perceived surface.

\section{Spatial context}

Besides temporal adaptation effects, another frequently studied concept in vision is the influence of spatial context on perception. An example is chromatic induction in the perception of colour (e.g. Cao and Shevell 2005; Shevell and Wei 2000; Webster et al. 2002). In these studies, observers had to judge the perceived colour of test surfaces, while simultaneously viewing inducing backgrounds composed of different colours. Two different types of induction were demonstrated: contrast and assimilation. Contrast occurs when the perceived appearance of the test shifts away from the appearance of the inducing stimulus; assimilation occurs when the appearance of the test shifts towards the appearance of the inducer. It has been proposed that factors like spatial frequency (Smith et al. 2001), luminance contrast, width of the inducing ring and receptive-field organization (Cao and Shevell 2005) play an important role in the transition from chromatic assimilation to chromatic contrast.

In the haptic modality, spatial context can be described as the interaction of information simultaneously received from different parts of the hand or, more specifically, from different fingers. Using sandpaper as stimuli, Dorsch et al. (2001) showed that when two fingers scanned surfaces with different grit numbers, the grit number presented to the non-attended finger had no effect on perceived roughness with the attended finger. This result suggests that there is no interaction between signals from different fingers and, hence, no influence of the spatial context on roughness perception.

However, studies using magnetoencephalography (MEG) and microelectrode recordings demonstrated interactions between finger representations. Researchers found multi-finger or wide-field receptive fields, which cover more than one finger, in area 1 neurons of the primary somatosensory cortex as well as in the medial part of the cortical finger region (Biermann et al. 1998; Forss et al. 1995; Iwamura et al. 1983). In general, these studies found an inhibition effect of the cerebral signal when multiple fingers were stimulated by mechanical stimulations of 
high-level intensities. When using low-level stimulations, which are more representative of the signals that we receive from our natural environment, the input from two fingers produced additive or facilitatory interactions in the early component of the cerebral potential (Gandevia et al. 1983). Furthermore, a number of studies have demonstrated the existence of multi-finger receptive fields in areas of the second somatosensory cortex (Fitzgerald et al. 2006; Sinclair and Burton 1993). Together, these results suggest that spatial context should influence perception. The fact that no interactions were found in the experiment by Dorsch et al. (2001) could be due to their use of sandpaper as stimuli. As argued by Hollins et al. (2006), the use of abrasive papers can cause damage to the skin and therefore alter the biophysical response to the stimuli. Consequently, it is not possible to draw consistent conclusions about the influences of spatial context on haptic roughness perception.

Our second experiment was designed to shed new light on spatial contextual influences in the haptic perception of roughness. The integration of information received from different fingers when scanning textured surfaces was investigated. Subjects were asked to discriminate between successively scanned surfaces while an adjacent finger was simultaneously scanning another surface varying in roughness. Based on neurophysiological studies concerning multi-finger receptive fields, we hypothesize that roughness information received from adjacent fingers will cause interaction effects. These effects will likely resemble chromatic assimilation rather than contrast effects, as Gandevia et al. (1983) has shown that low-level stimuli produces additive interactions.

\section{General methods}

\section{Subjects}

Ten subjects (six female and four male, mean age 20.2 years) participated in both experiments. To control for order effects, five subjects performed Experiment 1 before Experiment 2, and the other five participated in the reverse order. Nine subjects were strongly right-handed, and one was strongly left-handed, as established by Coren's handedness questionnaire (Coren 1993). All subjects were experimentally naïve and were paid for their participation. Before starting the first experiment, they provided written informed consent.

\section{Stimuli}

The stimuli used in both experiments were a set of embossed dot surfaces. The dot patterns were embossed on paper (weight $160 \mathrm{~g} / \mathrm{m}^{2}$ ) using an Emprint Braille Embosser (ViewPlus Technologies, emboss printing resolution 20 dots/inch). Each pattern was then pasted on $2.6 \mathrm{~mm}$-thick cardboard. It was necessary that the physical characteristics of the dots, especially the height profile, remained constant during the experiment. Therefore, every new condition of every subject began with a new stimulus set.

A total of 12 different patterns were constructed. Each pattern consisted of a specific part of $5.08 \times 5.08 \mathrm{~mm}$. This part was repeated 5 times in the horizontal and 20 times in the vertical direction, resulting in a $25.4 \mathrm{~mm}$ wide and $101.6 \mathrm{~mm}$ long stimulus pattern. One such specific part was composed of dots (height $0.4 \mathrm{~mm}$, diameter $0.8 \mathrm{~mm}$ ) placed in the centres of a regular $4 \times 4$ grid (Fig. 1a). The sequence of the 12 different patterns, with decreasing dot densities, was constructed by repeatedly removing one random dot from the previous specific part in the sequence (Fig. 1b). For each pattern, the average centre-to-centre distance between dots was calculated by taking the square root of the inverse dot-density. Consequently, for the complete stimulus set, the average distances between dots ranged from 1.27 to $2.27 \mathrm{~mm}$.

As previously demonstrated for embossed dot surfaces, dot spacing correlates with the subjective roughness of those surfaces (e.g. Chapman et al. 2002; Connor et al. 1990; Connor and Johnson 1992). These studies have shown a near linear increase in perceived roughness magnitude with increasing dot distances up to $3 \mathrm{~mm}$ (Connor et al. 1990; Connor and Johnson 1992) and in some studies for even larger distances (Chapman et al. 2002). Connor et al. (1990) found that the increase in perceived roughness for these dot distances is preserved for dots with varying diameter. This relationship is assumed to hold in the present study, in which the average distances between dots are smaller than these aforementioned maxima (see Fig. 1c). To find support for this assumption, a pilot study was performed in which blindfolded subjects had to order the patterns from the current study according to their perceived roughness. This pilot study demonstrated an increase in perceived roughness with increasing average distances between dots. Therefore, in the present study, a stimulus with a small average distance between dots was marked as a smooth stimulus, while a stimulus with a large average distance was marked as a rough stimulus.

\section{Experiment 1: temporal context}

This first experiment investigates the influence of temporal context on the haptic perception of roughness. The effect of two different adaptation levels (i.e. rough and smooth) on the perceived roughness of a subsequently scanned surface was studied. 
Fig. 1 a Representation of how a specific part of a particular pattern was constructed. b The sequence of the stimulus patterns used in this study. Note that only $3 \times 10$ repetitions of the specific part are shown, while a complete pattern consisted of $5 \times 20$ repetitions. Not on scale. c This figure represents the patterns according to the corresponding average distance between dots. An increase in the average distance is assumed to correspond to an increase in the perceived roughness of the surface
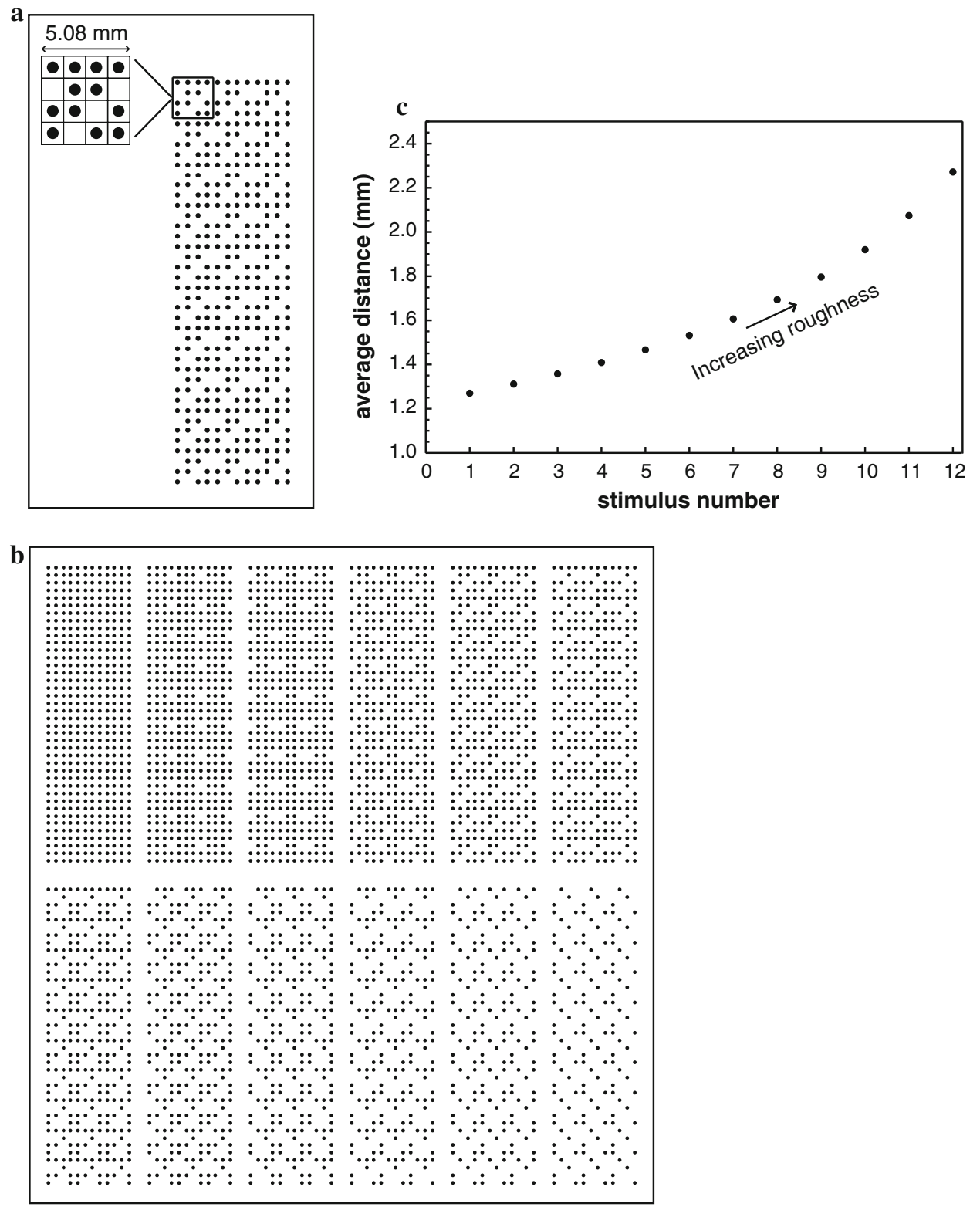

\section{Conditions}

The experiment included two adaptation conditions and one control condition. In the "rough adaptation condition", subjects first adapted their index finger to a rough stimulus. Then, they were asked to discriminate between the roughness of a stimulus perceived with the adapted index finger and the roughness of another surface perceived with the non-adapted middle finger of the same hand. In the "smooth adaptation condition", the index finger was adapted to a smooth stimulus before the test phase. In the control condition, the test phase was not preceded by adaptation. The rough and smooth adaptation stimuli had average distances between dots of 2.27 and $1.27 \mathrm{~mm}$, respectively. As much as 11 test stimuli with average dot distances ranging from 1.31 to $2.27 \mathrm{~mm}$ and a reference stimulus of $1.61 \mathrm{~mm}$ average distance were used. During the test phase, each combination of a particular test and reference stimulus was repeated ten times. Consequently, each condition consisted of 110 trials, resulting in a total of 330 trials for the entire experiment. The two adaptation conditions lasted approximately $60 \mathrm{~min}$ each, while the control condition was performed within $30 \mathrm{~min}$. Subjects performed the three conditions on different days and in a counterbalanced order.

\section{Procedure}

Before the experiment started, the participants were blindfolded to prevent them from using visual information 
during the experiment. About $25 \mathrm{~cm}$ in front of the subject, a cardboard framework was fixed on the table. The stimuli could be placed in between the borders of this framework in such a way that they could not move when the subject explored them (see Fig. 2). The subjects were instructed to apply a comfortable level of downward force with the tips of the index and middle finger and to move with a comfortable speed. The required movement was a forward and backward movement over the stimulus surfaces. Within a couple of practice trials, this movement pattern was trained. The subjects were asked to keep this movement pattern as constant as possible during the experiment. If large deviations from the trained movements were observed, instructions were given to correct the movement. Once the preferred movement pattern was achieved and the instructions were clear, the experimental runs started.

With regard to the two adaptation conditions, the first trial was preceded by a pre-adaptation period of $60 \mathrm{~s}$. In this way, a baseline level of adaptation was established before the first test trial started. All other test trials were preceded by an adaptation phase of $20 \mathrm{~s}$. To start the adaptation, the participant lowered the tip of the index finger of his/her dominant hand onto the stimulus surface and moved it over the stimulus surface, as trained during the practice trials (Fig. 2a). At the end of the adaptation period, the experimenter gave a vocal signal to stop adaptation and to move towards the next two stimuli. One of these stimuli was for the index finger, and the other one was for the middle finger (Fig. 2b). The position of the test and reference stimuli (i.e. under index or middle finger) as well as the order of the different test-reference combinations for each trial were randomized.

Next, the participant simultaneously moved the index and middle finger forward and backward over the stimuli. Immediately after completing the exploration, a two- alternative forced-choice (2AFC) task was conducted; the subject had to say which of the two stimuli, i.e. the stimulus scanned with the index or middle finger, felt rougher. After the response, the next adaptation phase began. The control condition proceeded in the same way, except that there was no adaptation phase. Hence, the control condition consisted of only the 2AFC task, which was conducted in the same way as during the adaptation conditions.

\section{Analysis}

The difference between the dot distance values of the stimuli scanned with the index and middle finger was used as the independent variable. For all subjects and conditions, we calculated for each of these differences the fraction with which the subject selected the stimulus scanned with the index finger as being rougher compared to the middle finger stimulus. A cumulative Gaussian distribution $(f)$ as function of the dot distance differences $(x)$ was fitted to the data using the following equation:

$f(x)=\frac{1}{2}\left(1+\operatorname{erf}\left(\frac{x-\mu}{\sigma \sqrt{2}}\right)\right)$,

where $\sigma$ is a measure of the discrimination threshold, indicating the shallowness of the curve, and $\mu$ is the observer's point of subjective equality (PSE), representing the location of the curve relative to the point of equal physical roughness. The discrimination threshold reveals the sensitivity of the subjects to perceived roughness differences within the experiment. The PSE corresponds to the physical roughness difference between the stimulus presented to the index finger and the stimulus presented to the middle finger that are on average judged as being equal. A shift of the curve in the horizontal direction can occur when subjects systematically underestimate or
Fig. 2 a Index finger moving over the adaptation stimulus. The other two stimuli are a test and the reference stimuli for the test phase. b Index and middle fingers moving over the two stimuli during the test phase. The arrow indicates which stimuli have to be compared
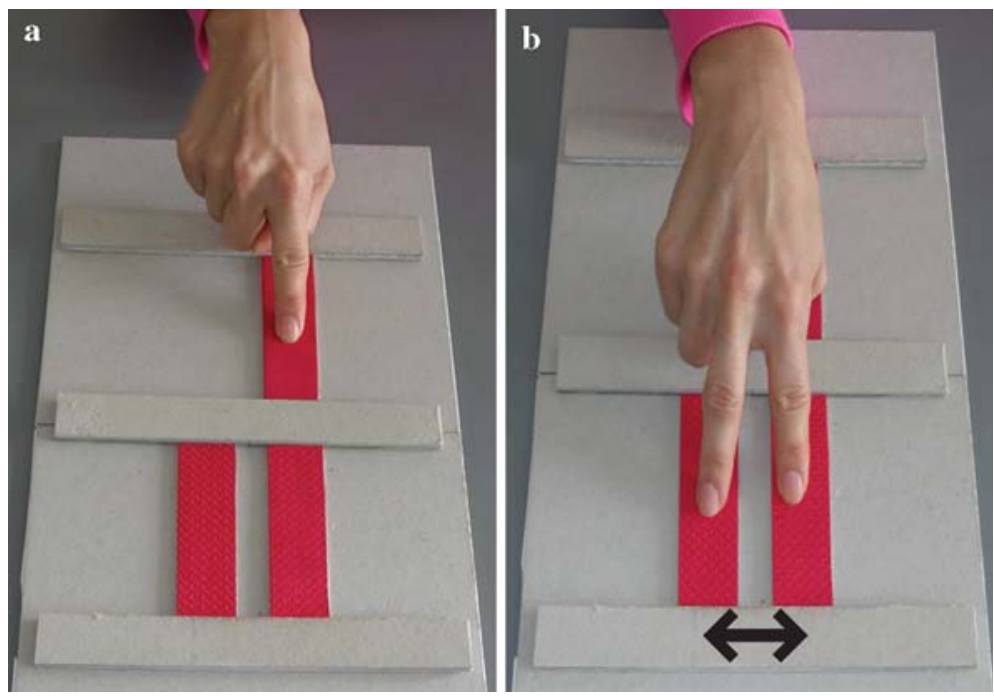
overestimate the roughness of the stimulus scanned with the index finger as compared to the stimulus scanned with the middle finger. Comparison of PSEs (i.e. the shift of the curves) under different conditions can reveal a possible effect of adaptation. Examples of this fitting procedure are shown in Fig. 3.

To compare the effects of the different adaptation conditions on the PSE, a repeated measures ANOVA was performed, with condition as the within-subject factor. Furthermore, the same significance test was performed with the measured thresholds to determine if there was an adaptation effect on discrimination ability. If significant overall effects were found, a paired comparison post hoc test was performed to reveal pairwise differences. To correct for multiple comparisons, a Bonferroni adjustment was done. For all statistic tests, $\alpha$ was set at $5 \%$.

\section{Results}

Figure 4 presents the average results for the effect of texture adaptation on roughness perception. The repeated measures ANOVA revealed a significant main effect of adaptation condition $\left(F_{2,18}=23.2, P<0.001\right)$. As shown in the figure, adaptation to a smooth or rough stimulus resulted in negative and positive biases, respectively. The average PSEs for the two adaptation conditions were $-0.09 \mathrm{~mm}$ and $0.15 \mathrm{~mm}$, corresponding to 5.3 and $9 \%$ of the average distance between dots of the reference stimulus. The negative bias indicates that the perceived roughness of the stimulus scanned with the index finger increased after adapting the index finger to a smooth stimulus. On the other hand, the positive bias shows that adapting the index finger to a rough stimulus resulted in a decrease of the perceived roughness of a subsequently scanned stimulus. Pairwise comparison showed that this difference between the two adaptation conditions was significant at $P<0.005$. Furthermore, significant

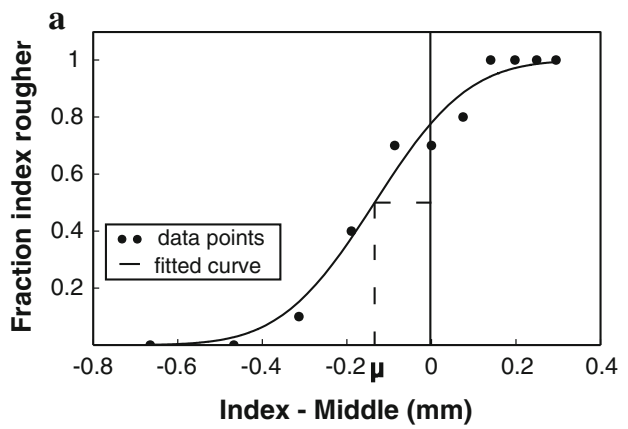

Fig. 3 Two examples of a psychometric function fitted to the data of a single subject. A data point shows, for a particular roughness difference, the fraction of times the subject judged the stimulus presented to the index finger as rougher than the stimulus presented to

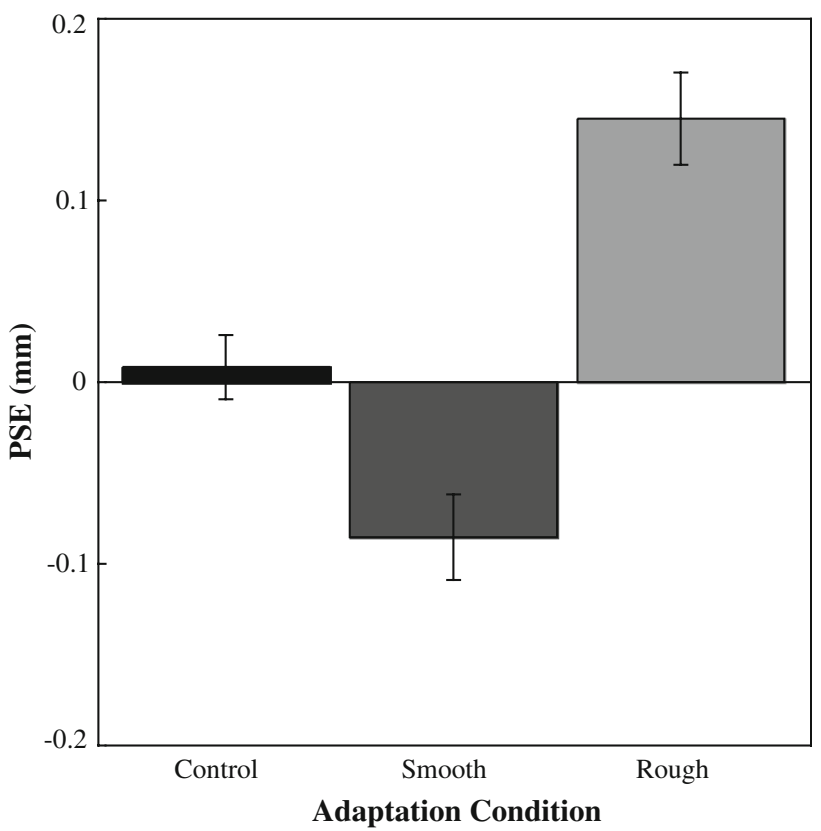

Fig. 4 Mean points of subjective equality (PSE) for the different adaptation conditions. The error bars represent the standard errors of the mean

differences between the two adaptation conditions and the control condition were found, with $P<0.05$ and $P<0.001$ for the smooth and rough conditions, respectively.

To explore the data in more detail, the complete data set was divided into a part in which the reference stimulus was scanned with the index finger and a part in which the reference stimulus was scanned with the middle finger. A 3 (condition) $\times 2$ (position) repeated measures ANOVA was performed on this data set, to test for significant effects of stimulus position. However, the effect of position was not significant $\left(F_{1,9}=2.39, P=0.16\right)$. Therefore, there was no need to distinguish between the locations of the reference stimulus in the data analysis.

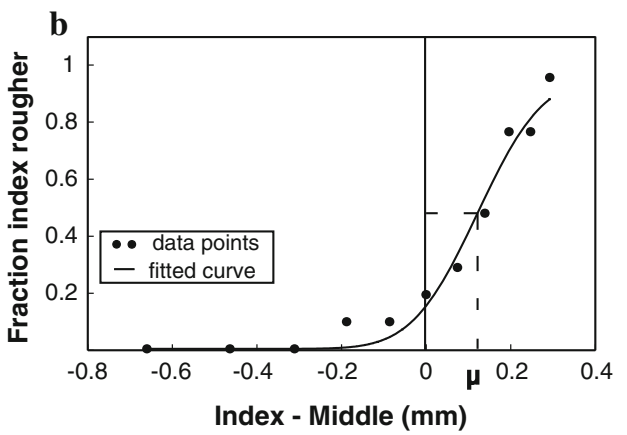

the middle finger. The dashed lines indicate the $\mu$ values. The figures depict a smooth (a) and a rough (b) adaptation stimulus condition with negative and positive PSE, respectively 


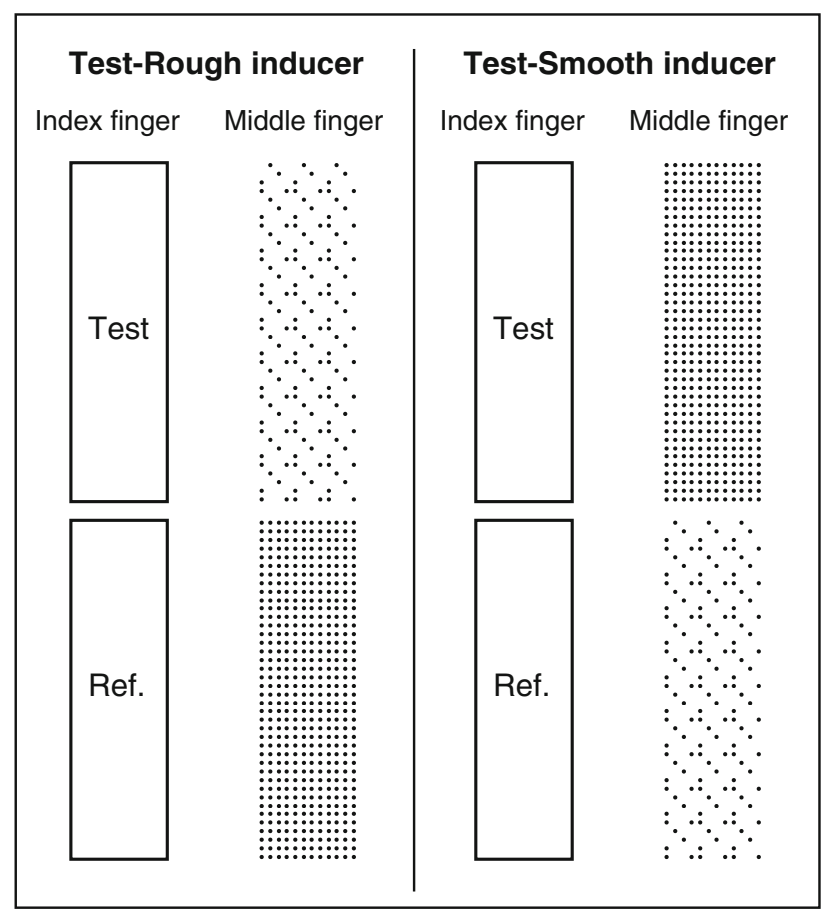

Fig. 5 Representation of the two inducer conditions: left the testrough inducer condition; right the test-smooth inducer condition

The average discrimination thresholds for the control, rough adaptation and smooth adaptation conditions were 0.15 (SD 0.01), 0.27 (SD 0.07) and 0.20 mm (SD 0.03), respectively. A repeated measures ANOVA showed no significant main effect of condition on these discrimination thresholds $\left(F_{2,18}=2.12, P=0.15\right)$.

\section{Experiment 2: spatial context}

The second experiment investigated the spatial contextual influences on roughness perception. A rough or smooth inducer stimulus was felt with one finger and its effect on roughness perception with an adjacent finger was examined.

\section{Conditions}

Figure 5 shows a representation of the two conditions. In the "test-rough inducer condition", the index finger explored a test stimulus while the middle finger of the same hand scanned a rough surface at the same time ("testrough pair"). Next, the index finger explored a reference stimulus, while the middle finger scanned a smooth surface at the same time ("reference-smooth pair"). The perceived roughness of the test stimulus from the "test-rough pair" was compared to the perceived roughness of the reference stimulus from the "reference-smooth pair".
In the "test-smooth inducer condition", the reverse was presented; the test stimulus was coupled with a smooth surface ("test-smooth pair") and compared to the reference stimulus coupled with a rough surface ("referencerough pair"). By comparing the two conditions, the effect of the inducer stimulus on the perceived roughness of the adjacent finger can be revealed. The rough and smooth inducer stimuli had the same average distance between dots as the rough and smooth adaptation stimuli from Experiment 1 . The same test and reference stimuli were also used.

The two inducer conditions were mixed within the same run, and the trials from the two different conditions were performed in a random order. The presentation order of the test and reference stimuli was also randomized; that is, the reference stimulus was felt before the test stimulus in some trials and presented in reverse order in other trials. Each condition contained 110 trials, resulting in 220 trials for the complete experiment. The experiment was performed within a single session lasting approximately $75 \mathrm{~min}$.

\section{Procedure}

The instructions for moving the fingers over the stimuli were the same as for Experiment 1; again, some practice trials preceded the experiment. First, the participant lowered the tips of the index and middle fingers onto the nearest two surfaces (see Fig. 6a). The index finger was placed onto the stimulus on the left and the middle finger onto the stimulus on the right (for the left-handed subject, the stimuli were reversed such that for the left- and righthanded subjects the same stimuli were scanned with the index and middle finger). Subsequently, participants simultaneously performed two forward and backward movements over the stimuli with the index and middle fingers (identical to the Experiment 1 test trials). Then, they raised their hand, replaced it towards the second pair, and repeated the exploration movement (Fig. 6b). Immediately after completing the second exploration, a 2AFC task was conducted; the subjects had to compare the two stimuli scanned with the index finger and say which of the two was perceived as rougher. After responding, the experimenter replaced the surfaces and another trial began.

Analysis

The difference between the average dot distances of the test and reference stimuli was used as the independent variable. For all subjects and both conditions, we calculated for each of these differences the fraction with which the subject responded that the test surface felt rougher compared to the reference surface. The same data fitting procedure as in Experiment 1 was used. To compare the effects of the inducer stimulus on the PSE and on the discrimination 
Fig. 6 a Index and middle fingers moving over the first two stimuli during a trial from Experiment 2. b Movement performed during the second part of the trial. The arrow indicates which stimuli have to be compared
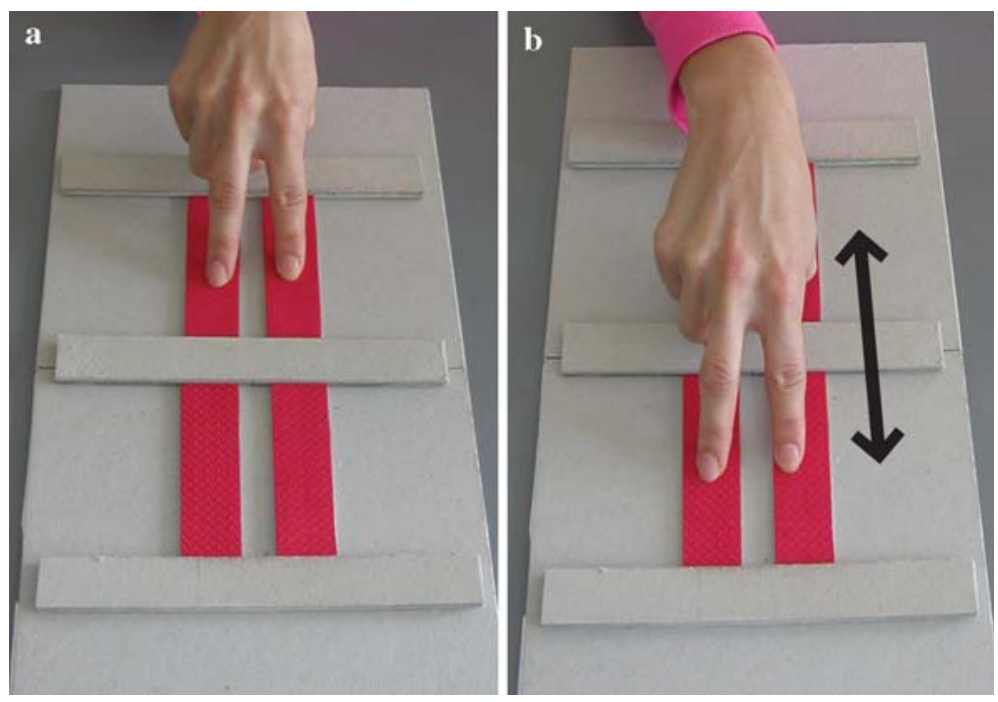

thresholds, repeated measures ANOVAs were performed, with condition as the within-subject factor.

\section{Results}

Figure 7 shows the effect of the roughness of an inducer stimulus scanned with the middle finger on the perceived roughness of a stimulus scanned simultaneously with the index finger of the same hand. The average PSEs were

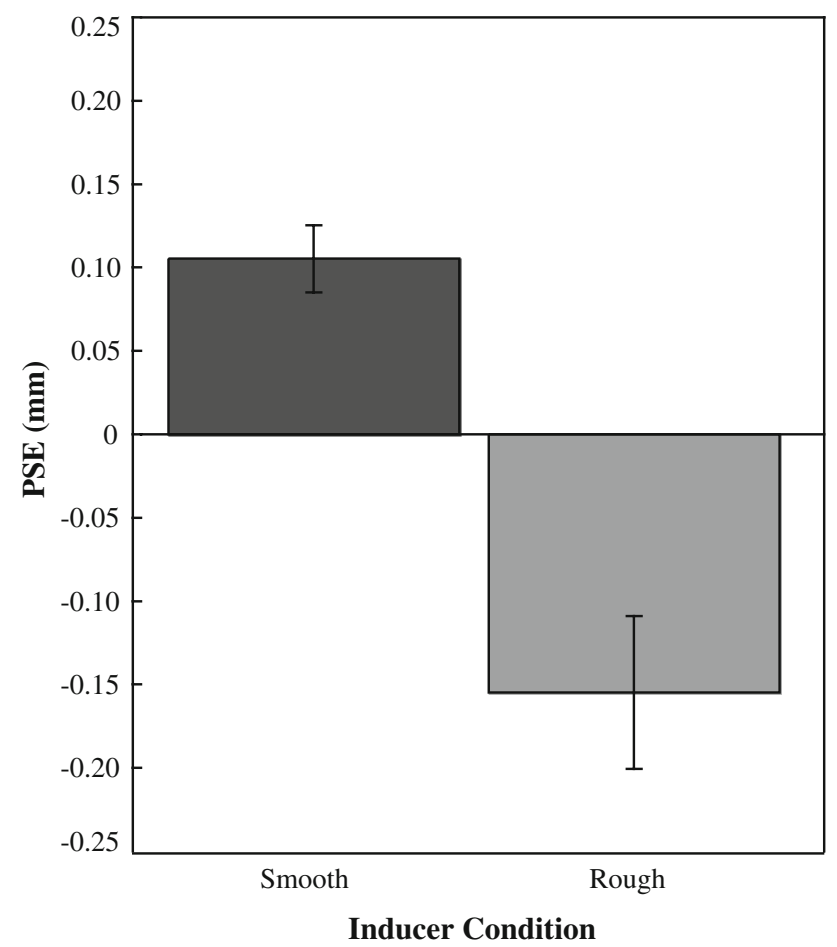

Fig. 7 Mean points of subjective equality ( $P S E$ ) for different inducer conditions. The error bars are the standard errors of the mean
$0.10 \mathrm{~mm}$ and $-0.15 \mathrm{~mm}$ for the smooth and rough inducer conditions, respectively. This corresponds to 6.4 and $9.2 \%$ of the average distance between dots of the reference stimulus. The repeated measures ANOVA showed a significant difference between the smooth and rough inducer conditions $\left(F_{1,9}=16.5, P<0.005\right)$. As seen in the Fig. 7 , a smooth inducer stimulus on the middle finger caused a positive bias, meaning that the perceived roughness of the stimulus scanned simultaneously with the index finger decreased. The negative bias in the rough inducer condition indicates that the perceived roughness of the stimulus felt with the index finger increased when a rough surface was scanned simultaneously with the middle finger. These results show that for both conditions, the perceived roughness of the stimulus felt with the index finger shifted toward the roughness of the inducer stimulus. The average discrimination thresholds were 0.10 (SD 0.01) and $0.16 \mathrm{~mm}$ (SD 0.04) for the smooth and rough conditions, respectively. As in Experiment 1, the difference between these discrimination thresholds was not significant $\left(F_{1,9}=2.85, P=0.13\right)$.

\section{Discussion}

The present study investigated the influences of temporal and spatial context on haptic roughness perception. It was found that temporal adaptation to a roughly (smoothly) textured surface resulted in a decrease (increase) of the perceived roughness of a subsequently scanned surface. Furthermore, the spatial context exerted its influence by shifting the perceived roughness of a surface towards the roughness of a simultaneously scanned inducer stimulus. These results are important for understanding the mechanisms involved in haptic roughness perception. 
Temporal effects

In the first experiment, after scanning a surface for a prolonged period of time with the index finger, participants had to discriminate between the roughness of a surface scanned with the adapted finger and the roughness of a surface scanned with an unadapted adjacent finger. The results showed a temporal context effect. Adaptation to a rough surface decreased perception of a surface scanned subsequently with the adapted finger. On the other hand, adaptation to a smoothly textured surface increased the perceived roughness of subsequently scanned surfaces.

These texture adaptation effects are in accordance with results from previous studies showing adaptation after effects in the haptic modality (Lederman et al. 1982; Van der Horst et al. 2008; Vogels et al. 2001). These studies show that adaptation to a physical dimension changes the perception of a subsequently perceived stimulus. This change is in the opposite direction to that of the adapting stimulus. They also proposed that higher levels of processing are involved.

The fact that rough and smooth adaptation resulted in opposite effects indicates that the process involved in texture adaptation is not simply a peripheral effect. If that were the case, then scanning either a smooth or rough surface for a prolonged period of time should cause the peripheral neurons to be over-stimulated, with the smooth surface producing relatively less over-stimulation. Therefore, adaptation to a smooth surface should show an effect in the same direction as adaptation to a rough surface, with only a smaller magnitude of that effect. Moreover, if it were a peripheral effect, then adaptation to a rough stimulus should disturb discrimination performance more than adaptation to a smooth stimulus. However, no significant difference between the discrimination thresholds measured in the three conditions was found, indicating that the ability of discrimination is not disturbed by adaptation. Therefore, a peripheral over-stimulation mechanism could not be the origin for the presented effect. Consequently, these findings suggest that the texture adaptation effect occurs at a higher level of processing.

Another relevant point is that adapting the index finger to a surface may modify the roughness not only of the stimulus subsequently scanned with the index finger, but also of the comparison stimulus scanned with the middle finger. Furthermore, interaction effects are possible between the signals received from the index and middle fingers when they were simultaneously scanning a stimulus during the test phase. These confounding factors can result in a decrease of the biases. However, the present experiment revealed highly significant effects regardless of these confounding factors. This shows that the presented effects are quite robust.
The results of this study indicate that the spatial pattern is already processed further before the effect is manifested. The neurons that code for roughness magnitude likely adapt to the roughness of the scanned surface. This finding can be explained by structures of the receptive fields of neurons in the somatosensory cortex. As stated in "Introduction", it has been shown that cells in the somatosensory and visual cortex have comparable receptive field structures (Dicarlo et al. 1998; Hubel and Wiesel 1962). Visual cortex cells show strong adaptation effects (e.g. Blakemore et al. 1973; Carandini et al. 1997; Dragoi et al. 2000; Jones and Palmer 1987). Therefore, we suggest that if the somatosensory cells are stimulated with appropriate stimuli, they should show comparable adaptation effects, and texture adaptation effects on roughness perception should be found. This was indeed the case. Furthermore, the correlation between our findings and those of Dicarlo et al. (1998) implies that our stimuli, random dot patterns with relatively large distances between dots, are appropriate stimuli for these adaptation neurons. Probably, these neurons do not respond in the same way to patterns with smaller dot distances, as those used by Hollins et al. (2006), or these patterns are too weak to cause significant adaptation effects. In general, the texture adaptation effect presented here supports the argument that visual and haptic modalities have similar structures and functions.

\section{Spatial effects}

The second experiment was based on the spatial influences of the context during haptic roughness perception. Participants had to discriminate between the roughness of two successively scanned surfaces while scanning a smooth or rough surface with an adjacent finger. The results showed that the perceived the roughness of a surface scanned with the index finger changed in the direction of the inducer stimulus; e.g. a smooth surface felt smoother (rougher) when perceived in the context of a smooth (rough) stimulus.

This spatial contextual effect supports findings from neurophysiological studies, which show that integration of information received from different fingers occurs along the processing pathway (Biermann et al. 1998; Forss et al. 1995; Gandevia et al. 1983; Iwamura et al. 1983). In addition, the present results show that this integration effect is also visible when natural stimuli are used and explored actively. This contrasts with the results from the study by Dorsch et al. (2001), where exploration of abrasive papers with two fingers did not result in any integration effects; however, the use of abrasive papers could have influenced their result.

The shift in perceived roughness of the adjacent stimulus resembles the visual assimilation effect, which also 
occurs when the appearance of the test shifts towards the appearance of the inducer (e.g. Cao and Shevell 2005; Smith et al. 2001). Some neural mechanisms are proposed to account for observed assimilation effects in the visual domain (Cao and Shevell 2005; De Weert and Van Kruysbergen 1997; Shevell and Wei 2000). One suggested mechanism is spatial averaging of the neural signals in combination with the size of the receptive fields. During presentation of stimuli composed of a test and inducer rings, only the stimuli containing smaller inducer rings results in assimilation. It has been proposed that if neural spatial summation occurs in the centres of the centre-surround receptive fields and the inducer rings are small enough to fall within the centre of the receptive field that also registers the test stimulus, then an assimilation effect will occur. The spatial contextual effect in haptic roughness perception could be explained by a comparable mechanism in which signals from the index finger and from the inducer middle finger both fall within the centre of the same receptive field, producing the assimilation effect. These receptive fields could be the multi-finger receptive fields that were found at the level of the somatosensory cortex where integration of information received from different fingers occurs (Biermann et al. 1998; Fitzgerald et al. 2006; Forss et al. 1995; Iwamura et al. 1983; Sinclair and Burton 1993).

\section{Conclusion}

The results from the present two experiments show strong effects of context during haptic perception of roughness. Temporal adaptation causes roughness perception to shift away from the roughness of the adaptation stimulus (i.e. contrast after effect), while simultaneous stimulation of the fingers causes the perception to shift towards the adjacent stimulus (i.e. assimilation effect). Although these effects seem contradictory, we can explain them using comparable mechanisms. We suggest that these effects do not manifest themselves at a lower, peripheral level of processing, but rather that high-level mechanisms are involved. Structures of the cortical receptive fields are proposed as an explanation for the temporal as well as spatial contextual effects. The analogies with comparable effects in the visual system emphasize the similarities of the different modalities.

Acknowledgment This research was supported by a grant from The Netherlands Organization for Scientific Research (NWO).

Open Access This article is distributed under the terms of the Creative Commons Attribution Noncommercial License which permits any noncommercial use, distribution, and reproduction in any medium, provided the original author(s) and source are credited.

\section{References}

Adelson EH (1993) Perceptual organization and the judgment of brightness. Science 262:2042-2044

Biermann K, Schmitz F, Witte OW, Konczak J, Freund HJ, Schnitzler A (1998) Interaction of finger representation in the human first somatosensory cortex: a neuromagnetic study. Neurosci Lett 251:13-16

Blake DT, Hsiao SS, Johnson KO (1997) Neural coding mechanisms in tactile pattern recognition: The relative contributions of slowly and rapidly adapting mechanoreceptors to perceived roughness. J Neurosci 17:7480-7489

Blakemore C, Muncey JPJ, Ridley RM (1973) Stimulus specificity in the human visual system. Vision Res 13:1915-1931

Cao D, Shevell SK (2005) Chromatic assimilation: spread light or neural mechanism? Vision Res 45:1031-1045

Carandini M, Barlow HB, O'Keefe LP, Poirson AB, Anthony Movshon J (1997) Adaptation to contingencies in macaque primary visual cortex. Philos Trans R Soc Lond B Biol Sci 352:1149-1154

Chapman CE, Tremblay F, Jiang W, Belingard L, Meftah EM (2002) Central neural mechanisms contributing to the perception of tactile roughness. Behav Brain Res 135:225-233

Connor CE, Hsiao SS, Phillips JR, Johnson KO (1990) Tactile roughness: neural codes that account for psychophysical magnitude estimates. J Neurosci 10:3823-3836

Connor CE, Johnson KO (1992) Neural coding of tactile texture: comparison of spatial and temporal mechanisms for roughness perception. J Neurosci 12:3414-3426

Coren S (1993) The left-hander syndrome. Vintage Books, New York

De Weert CMM, Van Kruysbergen NAWH (1997) Assimilation: central and peripheral effects. Perception 26:1217-1224

Dicarlo JJ, Johnson KO, Hsiao SS (1998) Structure of receptive fields in area $3 \mathrm{~b}$ of primary somatosensory cortex in the alert monkey. J Neurosci 18:2626-2645

Dorsch AK, Hsiao SS, Johnson KO, Yoshioka T (2001) Tactile attention: subjective magnitude estimates of roughness using one or two fingers. In: Society for Neuroscience Abstracts, vol 27

Dragoi V, Sharma J, Sur M (2000) Adaptation-induced plasticity of orientation tuning in adult visual cortex. Neuron 28:287-298

Fitzgerald PJ, Lane JW, Thakur PH, Hsiao SS (2006) Receptive field (RF) properties of the macaque second somatosensory cortex: RF size, shape, and somatotopic organization. J Neurosci 26:6485-6495

Forss N, Jousmaki V, Hari R (1995) Interaction between afferent input from fingers in human somatosensory cortex. Brain Res 685:68-76

Gandevia SC, Burke D, McKeon BB (1983) Convergence in the somatosensory pathway between cutaneous afferents from the index and middle fingers in man. Exp Brain Res 50:415-425

Hollins M, Bensmaïa SJ, Washburn S (2001) Vibrotactile adaptation impairs discrimination of fine, but not coarse, textures. Somatosens Mot Res 18:253-262

Hollins M, Lorenz F, Harper D (2006) Somatosensory coding of roughness: the effect of texture adaptation in direct and indirect touch. J Neurosci 26:5582-5588

Hubel DH, Wiesel TN (1962) Receptive fields, binocular interaction and functional architecture in the cat's visual cortex. J Physiol 160:106-154

Iwamura Y, Tanaka M, Sakamoto M, Hikosaka O (1983) Converging patterns of finger representation and complex response properties of neurons in area 1 of the first somatosensory cortex of the conscious monkey. Exp Brain Res 51:327-337

Jones JP, Palmer LA (1987) The two-dimensional spatial structure of simple receptive fields in cat striate cortex. J Neurophysiol 58:1187-1211 
Lederman SJ (1981) The perception of surface roughness by active and passive touch. Bull Psychon Soc 18:253-255

Lederman SJ (1983) Tactual roughness perception: spatial and temporal determinants. Can J Psychol 37:498-511

Lederman SJ, Taylor MM (1972) Fingertip force, surface geometry, and the perception of roughness by active touch. Percept Psychophys 12:401-408

Lederman SJ, Loomis JM, Williams BA (1982) The role of vibration in the tactual perception of roughness. Percept Psychophys 32:109-116

Shevell SK, Wei J (2000) A central mechanism of chromatic contrast. Vision Res 40:3173-3180

Sinclair RJ, Burton H (1993) Neuronal activity in the second somatosensory cortex of monkeys (Macaca mulatta) during active touch of gratings. J Neurophysiol 70:331-350
Smith VC, Jin PQ, Pokorny J (2001) The role of spatial frequency in color induction. Vision Res 41:1007-1021

Van der Horst BJ, Duijndam MJA, Ketels MFM, Wilbers MTJM, Zwijsen SA, Kappers AML (2008) Intramanual and intermanual transfer of the curvature after effect. Exp Brain Res 187:491-496

Vogels IMLC, Kappers AML, Koenderink JJ (2001) Haptic aftereffect of successively touched curved surfaces. Acta Psychol 106:247-263

Ware C, Cowan WB (1982) Changes in perceived color due to chromatic interactions. Vision Res 22:1353-1362

Webster MA, Malkoc G, Bilson AC, Webster SM (2002) Color contrast and contextual influences on color appearance. J Vision 2:505-519 